\title{
Preface to the special issue for The Fifth Workshop on Formal Topology
}

\author{
THIERRY COQUAND \\ MARIA EMILIA MAIETTI \\ ERIK PALMGREN
}

This special issue of the Journal of Logic and Analysis hosts the proceedings of the Fifth Workshop on Formal Topology (5WFTop) held at Institute Mittag-Leffler, Djursholm, Sweden on June 8-10, 2015.

The workshop was administered and funded by Institute Mittag-Leffler, with additional financial support from the G.S. Magnusson Foundation and Japan Society for the Promotion of Sciences (JSPS), Core-to-Core Program and the University of Padova.

The workshop gathered about 30 researchers in formal topology, constructivism and related areas. A subtheme was modern developments in the theory of spreads and choice sequences, as well as its history.

Invited talks at the 5WFTop were given by Mark van Atten, Andrej Bauer, Francesco Ciraulo, Martín Escardó, Michael Fourman, Ieke Moerdijk, Joan Rand Moschovakis, Thomas Streicher, Anne S. Troelstra, Wim Veldman and Steven Vickers.

Contributed talks were given by Ulrich Berger, Thierry Coquand, Tatsuji Kawai, Maria Emilia Maietti, Per Martin-Löf, Yoshihiro Maruyama, Takako Nemoto, Paige North, Iosif Petrakis, Davide Rinaldi, Giovanni Sambin, Peter Schuster and Bas Spitters.

It was the first time that the Workshop on Formal Topology took place in Sweden and for the second time outside Italy. Indeed, previous workshops were held in Italy, in Padova (1997 and 2007), in Venice (2002), and in Ljubljana, Slovenia (2012).

It was in Sweden, and precisely in Stockholm, that the notion of formal topology was pioneered by Per Martin-Löf and Giovanni Sambin in a fruitful cooperation starting in 1984 and stretching over thirty years.

In few words, formal topology was introduced as a fully constructive approach to point-free topology. It was very much influenced by Brouwer's works on intuitionism.

Indeed, the study of the logical foundations of topology played an important role in the development of intuitionism. Early works by Brouwer on the theory of spreads and 
choice sequences, which was a subtheme of 5WFTop, were influencing much work in the area. A modernized form of his ideas is embodied in formal topology.

Furthermore, point-free methods, based on describing spaces by open neighborhoods and their covering relations, turned out also to be particularly useful in the various modern foundational approaches to constructive mathematics. These include topos theory, constructive set theory and type theory.

The peculiarity of formal topology is that of allowing a development of the classical theory of point-free spaces, known as Locale theory, in a fully constructive and predicative way.

A main purpose of the series of workshop on Formal Topology is to provide occasions to gather together prominent scholars developing different approaches to topology and in particular to point-free topology.

The 5WFTop workshop was followed by an open call for contributions to this special issue.

The contributions of this volume were selected and reviewed according to the rigorous standards of the journal. They represent work in formal topology, constructive topology according to Bishop, and interpretations of Brouwerian principles in terms of formal topology as well as more abstract considerations of spaces arising from topos theory.

We thank all the contributors to this volume, and the anonymous referees, for making it a valuable instrument for the future development on formal topology and related fields.

We are grateful to the Journal of Logic \& Analysis for the opportunity to publish a proceedings of 5WFTop in the form of this special issue.

Special issue editors:

Thierry Coquand

Maria Emilia Maietti

Erik Palmgren

Department of Computer Science and Electrical Engineering, University of Gothenburg, Sweden Department of Mathematics, University of Padova, Italy

Department of Mathematics, Stockholm University, Sweden

Thierry.Coquand@cse.gu.se, maietti@math.unipd.it, palmgren@math.su.se

Received: 10 June 2019

Journal of Logic \& Analysis 11:FT0 (2019) 